\title{
SCALAR CONSERVATION LAWS WITH CHARATHEODORY FLUX REVISITED
}

\author{
NikOLA KONATAR \\ University of Montenegro, Montenegro
}

\begin{abstract}
We introduce a new approach for dealing with scalar conservation laws with the flux discontinuous with respect to the space variable and merely continuous with respect to the state variable which employs a variant of the kinetic formulation. We use it to improve results about the existence of solutions for non-degenerate scalar conservation laws with Caratheodory flux under a variant of non-degeneracy conditions.
\end{abstract}

\section{INTRODUCTION}

Scalar conservation laws with discontinuous flux are a non-trivial generalization of scalar conservation law with smooth flux, and they describe different physical phenomena occurring in highly heterogeneous environments. Typical examples are flow in porous media, sedimentation processes, traffic flow, radar shape-from-shading problems, blood flow, gas flow in a variable duct etc. (see e.g. $[1,7,8,10]$ and references therein). Therefore, since the eighties (probably from [28]), scalar conservation laws with discontinuous flux are under intensive investigations.

Unlike the situation when the flux depends regularly on the space variable, the questions of existence and uniqueness of solution as well as existence of traces of entropy solutions are still open in the case when the flux is discontinuous. Indeed, in the classical work [13] one can find a comprehensive concept (well known entropy solutions) leading to the well posedness of scalar conservation laws with regular coefficients (continuously differentiable with respect to all the variables). It appeared that it is highly non-trivial to extend the concept to the case when the flux is discontinuous with respect to

2020 Mathematics Subject Classification. 35L65, 65M25.

Key words and phrases. Multidimensional scalar conservation law, discontinuous flux, existence, kinetic formulation, Caratheodory flux. 
the space variable and several interesting approaches appeared in $[2,3,12,22]$ most of them adapting the approach from [13]. Still, the question of existence and uniqueness of solution in the case when the flux is merely of bounded variation with respect to the space variable is still open.

The situation is even more complicated when the flux is merely continuous with respect to the state variable as in that case we do not have well-posedness. More precisely, in [21] one can find an example of a Cauchy problem for scalar conservation law with continuous flux admitting two different entropy solution with the same initial data. As for the existence, it holds even in the case when the flux is Caratheodory vector i.e. bounded variation with respect to the space variable and continuous with respect to the state variable under the so called non-degeneracy conditions ([23]). Under stronger non-degeneracy conditions, we shall improve and simplify the result from [23] (see also [20]).

In order to precisely formulate the problem, let us introduce the equation that we are going to consider:

$$
\partial_{t} u+\operatorname{div}_{\mathbf{x}} \mathfrak{f}(\mathbf{x} \cdot u)=0,
$$

where

$$
\text { A1. } \left.\mathfrak{f} \in B V\left(\mathbf{R}^{d} ; C\left(\mathbf{R}^{d}\right)\right) \text { and } \max _{\lambda \in[-M, M]}|\mathfrak{f}(\mathbf{x}, \lambda)| \in L_{l o c}^{1+\sigma}\left(\mathbf{R}^{d}\right)\right) \text {; }
$$

A2. it holds $\mathfrak{f}(\mathbf{x}, \lambda)=0$ for $\lambda \notin(a, b)$ for some $a, b \in \mathbf{R}$.

To proceed, denote

$$
\mathfrak{f}_{\delta}=\mathfrak{f} \star \frac{1}{\delta} \omega\left(\frac{\lambda}{\delta}\right)=\int \mathfrak{f}(\mathbf{x}, \eta) \frac{1}{\delta} \omega\left(\frac{\lambda-\eta}{\delta}\right) d \eta \text { and } \mathfrak{f}_{\delta}^{\prime}=\partial_{\lambda} \mathfrak{f}_{\delta} .
$$

We shall also assume the following non-degeneracy conditions.

DeFinition 1.1. We say that the flux $\mathfrak{f}$ satisfies the non-degeneracy conditions if there exists a non-negative function $\omega \in C_{c}^{\infty}(\mathbf{R})$ with total mass one and $p>1$ such that for any interval $I \subset \subset \mathbf{R}$, every $(\tau, \boldsymbol{\xi}) \in S^{d}$, where $S^{d}$ is a sphere in $\mathbf{R}^{d+1}$, and almost every $\mathbf{x} \in \mathbf{R}^{d}$, it holds for any $\delta=\delta\left(\delta_{0}\right)<\delta_{0}$ and some $\sigma\left(\delta_{0}\right) \rightarrow 0$ as $\delta_{0} \rightarrow 0$ :

$$
\liminf _{\delta_{0} \rightarrow 0}\left\|\frac{\left(\tau+\left\langle\mathfrak{f}_{\delta}^{\prime}(\mathbf{x}, \lambda), \boldsymbol{\xi}\right\rangle\right)\left(\tau+\left\langle\hat{f}_{\delta_{0}}^{\prime}(\mathbf{x}, \lambda), \boldsymbol{\xi}\right\rangle\right)}{\left|\tau+\left\langle\mathfrak{f}_{\delta_{0}}^{\prime}(\mathbf{x}, \lambda), \boldsymbol{\xi}\right\rangle\right|^{2}+\sigma\left(\delta_{0}\right)}-1\right\|_{L^{2}(I)}=0 .
$$

The previous definition essentially states that when the function $g(\lambda)=$ $\tau+\langle\mathfrak{f}(\mathbf{x}, \lambda), \boldsymbol{\xi}\rangle$ is differentiable, then its derivative must be different from zero on non-zero sets. If it is not differentiable, then we need to be able to choose the convolution kernel $\omega$ such that (1.2) holds (very informally speaking, for every $(\tau, \boldsymbol{\xi}) \in S^{d}$ and almost every $(t, \mathbf{x}) \in \mathbf{R}_{+}^{d+1}$, the inequality $\limsup _{\delta \rightarrow 0}\left|\tau+\left\langle f_{\delta_{0}}^{\prime}(\mathbf{x}, \lambda), \boldsymbol{\xi}\right\rangle\right| \geq c>0$ should hold almost everywhere for some fixed $c$, where $c$ can also be $\infty$ ). 
In [23], the non-degeneracy conditions are much simpler and it is required that for every $(\tau, \boldsymbol{\xi}) \in S^{d}$, where $S^{d}$ is a sphere in $\mathbf{R}^{d+1}$, and almost every $\mathbf{x} \in \mathbf{R}^{d}$, the mapping

$$
\lambda \mapsto \tau \lambda+\langle\mathfrak{f}(\mathbf{x}, \lambda), \boldsymbol{\xi}\rangle
$$

is non-constant on non-degenerate intervals.

It is not clear what the relation between (1.2) and (1.3) is, but if $\mathfrak{f}$ is continuously differentiable then (1.2) follows from (1.3) (see e.g. proof of [16, Theorem 3.4]).

The main result of the article is the following theorem.

Theorem 1.2. Equation (1.1) satisfying A1, A2, and the non-degeneracy conditions (1.2) augmented with the initial conditions $\left.u\right|_{t=0}=u_{0}(\mathbf{x})$ such that $a \leq u_{0} \leq b$ admits at least one weak solution.

The method of the proof consists in considering standard vanishing viscosity approximation and then proving that the family of approximate solutions is strongly $L_{l o c}^{1}$-precompact. The main tool for the convergence proof will be the reformulation of the problem in the kinetic formulation $([18])$, and then applying $\mathrm{H}$-measures $([9,26])$ and $\mathrm{H}$-distributions $([2,16])$ in order to obtain precompactness of the family of approximating solutions.

The paper is organized as follows. After the introduction, we recall the necessary notions from the $\mathrm{H}$-measures and $\mathrm{H}$-distributions. In the continuation of the section, we introduce the vanishing viscosity approximation and show that it admits a strongly converging subsequence in $L_{l o c}^{1}\left(\mathbf{R}^{+} \times \mathbf{R}^{d}\right)$.

\section{H-MEASURES AND H-Distributions}

Let us first recall basic notions concerning $\mathrm{H}$-measures and $\mathrm{H}$-distributions.

The first works concerning micro-local defect functionals are due to $\mathrm{P}$. Gerard ([9]) and L. Tartar ([26]) in the $L^{2}$-setting (independently of each other), and a generalization to the $L^{p}$-setting was achieved twenty years later in [2]. Further generalizations can be found in $[4,5,14,15,16,17,19,24,27]$

and in [25] where the connection between micro-local defect functionals and Young measures is discovered.

$\mathrm{H}$-measures, as well as any other micro-local defect functional describe the lack of $L^{p}$-strong convergence of the sequence $\left(u_{n}\right)$ for appropriate $p \geq 1$. More precisely, if the H-measure equals zero, then the sequence defining it strongly converges to zero in $L_{l o c}^{2}\left(\mathbf{R}^{d} ; \mathbf{R}^{m}\right)$ as well. It is a similar situation with the $\mathrm{H}$-distributions.

Here, we shall recall (probably) the most up-to date version of the $\mathrm{H}$ measures and H-distributions introduced in [16] on a new type of space. Let us introduce the notions that we are going to use here. We fix the notation 
by denoting by $s^{\prime}$ the conjugate of $s$ :

$$
\frac{1}{s}+\frac{1}{s^{\prime}}=1 \text {. }
$$

Next, let $\bar{s}>s>1$ and $r>1$ such that $1 / s^{\prime}+1 / \bar{s}=1 / r$. We denote $\|\cdot\|_{W^{(\bar{s}, s)}}$ the following functional

$$
\|\phi\|_{W^{(\bar{s}, s)}}=\sup _{\|\rho\|_{L^{s^{\prime}}\left(\mathbf{R}^{d+m}\right)}=1}\left(\int_{\mathbf{R}^{d}}\left\|\int_{\mathbf{R}^{m}} \rho(\mathbf{x}, \lambda) \phi(\mathbf{x}, \lambda, \boldsymbol{\xi}) d \lambda\right\|_{C^{d}\left(S^{d-1}\right)}^{r} d \mathbf{x}\right)^{1 / r}
$$

$$
\|\phi\|_{W_{0}^{(\bar{s}, s)}}=\sup _{\|\rho\|_{L^{s^{\prime}}\left(\mathbf{R}^{d+m}\right)}}\left(\int_{\mathbf{R}^{d}}\left\|\int_{\mathbf{R}^{m}} \rho(\mathbf{x}, \lambda) \phi(\mathbf{x}, \lambda, \boldsymbol{\xi}) d \lambda\right\|_{C\left(S^{d-1}\right)}^{r} d \mathbf{x}\right)^{1 / r} .
$$

for a measurable function $\phi$ for which (2.1) is finite.

The latter two functionals define the vector spaces introduced originally in $[16]$

$$
\begin{aligned}
& W^{(\bar{s}, s)}:=W^{(\bar{s}, s)}\left(\mathbf{R}^{d}, \mathbf{R}^{m}, S^{d-1}\right)=\left\{\phi:\|\phi\|_{W^{(\bar{s}, s)}}<\infty\right\} \\
& W_{0}^{(\bar{s}, s)}:=W_{0}^{(\bar{s}, s)}\left(\mathbf{R}^{d}, \mathbf{R}^{m}, S^{d-1}\right)=\left\{\phi:\|\phi\|_{W_{0}^{(\bar{s}, s)}}<\infty\right\} .
\end{aligned}
$$

In the sequel, we shall use the notation $W^{(\bar{s}, s)}$ and $W_{0}^{(\bar{s}, s)}$ whenever it is clear over which sets the space is defined. The space $W^{(\bar{s}, s)}$ has the following properties [17] (analogous properties hold for the space $W_{0}^{(\bar{s}, s)}$ ).

- By identifying two functions $\phi_{1}, \phi_{2} \in W^{(\bar{s}, s)}$ such that for almost every $\mathbf{x} \in \mathbf{R}^{d}$, every $\boldsymbol{\xi} \in S^{d-1}$, and almost every $\lambda \in \mathbf{R}^{m}$ it holds $\phi_{1}-\phi_{2}=0$, relation (2.1) defines a norm on $W^{(\bar{s}, s)}$.

- A function $\phi$ is zero in $W^{(\bar{s}, s)}$ if and only if for almost every $\mathbf{x} \in \mathbf{R}^{d}$, every $\boldsymbol{\xi} \in S^{d-1}$ the function $\lambda \rightarrow \phi(\mathbf{x}, \lambda, \boldsymbol{\xi})$ is zero a.e.

- The space $L^{\bar{s}}\left(\mathbf{R}^{d} ; L^{s}\left(\mathbf{R}^{m} ; C^{d}\left(S^{d-1}\right)\right)\right)$ is continuously embedded into $W^{(\bar{s}, s)}$ since

$$
\left\|\int_{\mathbf{R}^{m}} \rho(\mathbf{x}, \lambda) \phi(\mathbf{x}, \lambda, \boldsymbol{\xi}) d \lambda\right\|_{C^{d}\left(S^{d-1}\right)} \leq \int_{\mathbf{R}^{m}}|\rho(\mathbf{x}, \lambda)|\|\phi(\mathbf{x}, \lambda, \cdot)\|_{C^{d}\left(S^{d-1}\right)} d \lambda .
$$

- Let $\Omega_{\mathbf{x}}$ and $\Omega_{\lambda}$ be relatively compact sets in $\mathbf{R}^{d}$ and $\mathbf{R}^{m}$, respectively. Let $q>s$ and $1 / s^{\prime}+1 / \bar{s}=1 / q^{\prime}+1 / \bar{q}=1 / r$. Then the following continuous embedding holds ([17, Lemma 2.3])

$$
W^{(\bar{q}, q)}\left(\Omega_{x}, \Omega_{y}, S^{d-1}\right) \hookrightarrow W^{(\bar{s}, s)}\left(\Omega_{x}, \Omega_{y}, S^{d-1}\right) .
$$

- The set $C_{c}^{d}\left(\mathbf{R}^{d+m} \times S^{d-1}\right) \cap W^{(\bar{q}, q)}$ is dense in $W^{(\bar{q}, q)}$.

Now, we introduce a completition of the space $W^{\bar{s}, s}$ on which we can define the micro-local defect functional that we need. 
We call the $N$-partition, $N \in \mathbf{N}$, the decomposition of the space $\mathbf{R}^{d}$ on disjoint equal hyper-cubes with the edge parallel to the coordinate axis and of the length $1 / 2^{N}$ where the vertex of some of the hyper-cubes is at the origin.

Fix a relatively compact set $K \subset \subset \mathbf{R}^{m}$. Let $\Upsilon$ be a family of functions of the form

$$
\Upsilon=\left\{\sum_{j=1}^{\tilde{N}} \alpha_{j}(\boldsymbol{\xi}, \lambda) \chi_{j}^{N}(\mathbf{x}): \alpha_{j}^{N} \in C^{d}\left(S^{d-1} \times K\right), N, \tilde{N} \in \mathbf{N}\right\},
$$

where $\chi_{j}^{N}$ are characteristic functions of appropriate hypercubes from the $N$-partitions of $\mathbf{R}^{d}$.

By $\tilde{W}^{(\bar{s}, s)}$ we denote the closure of $\Upsilon$ in $W^{(\bar{s}, s)}$ :

$$
\tilde{W}^{(\bar{s}, s)}=C l_{\|\cdot\|_{W^{(\bar{s}, s)}}}(\Upsilon) .
$$

The following two theorems are slight modifications of the results from [17].

Theorem 2.1. Let $\left(u_{n}\right)$ be a bounded sequence in $L^{p}\left(\mathbf{R}^{d+m}\right), p \in(1,2]$, and let $\left(v_{n}\right)$ be a uniformly compactly supported bounded sequence of functions converging weakly to zero in $L^{q}\left(\mathbf{R}^{d}\right)$ for some (finite) $q>p^{\prime}$. Let $r \in(1, p)$ be such that $\frac{1}{p}+\frac{1}{r^{\prime}}+\frac{1}{q}=1$. Then, after passing to a subsequence (not relabeled), there exists a continuous bilinear functional $\mu$ on $L^{r}\left(\mathbf{R}^{d}\right) \otimes L^{p^{\prime}}\left(\mathbf{R}^{m} ; C^{d}\left(S^{d-1}\right)\right)$ such that for every $\phi \in L^{r}\left(\mathbf{R}^{d}\right)$ and $\psi \in L^{p^{\prime}}\left(\mathbf{R}^{m} ; C^{d}\left(S^{d-1}\right)\right)$, it holds

$$
\mu(\phi, \bar{\psi})=\lim _{n \rightarrow \infty} \int_{\mathbf{R}^{d+m}} \phi(\mathbf{x}) u_{n}(\mathbf{x}, \lambda) \overline{\mathcal{A}_{\psi(\lambda, \boldsymbol{\xi} /|\boldsymbol{\xi}|)}\left(v_{n}\right)(\mathbf{x})} d \mathbf{x} d \lambda,
$$

where $\mathcal{A}_{\psi(\lambda, \boldsymbol{\xi} /|\boldsymbol{\xi}|)}$ is the Fourier multiplier operator on $\mathbf{R}^{d}$ with the symbol $\psi(\lambda, \boldsymbol{\xi} /|\boldsymbol{\xi}|)$.

The functional $\mu$ is called the H-distribution corresponding to (sub)sequences (of) $\left(u_{n}\right)$ and $\left(v_{n}\right)$. It extends continuously to $\tilde{W}^{(\bar{s}, s)}$.

In the similar manner, we can introduce the completition of the space $W_{0}^{\bar{s}, s}$ simply be replacing $\|\cdot\|_{W^{\bar{s}, s}}$ by $\|\cdot\|_{W_{0}^{\bar{s}, s}}$ in (2.6). This is possible in the case when the sequence $\left(u_{n}\right)$ is bounded in $L^{p}\left(\mathbf{R}^{d+m}\right), p \geq 2$, since in this case we do not need to apply the Marcinkiewitz multiplier theorem but simply use the fact that a multiplier operator is bounded $L^{2} \rightarrow L^{2}$ mapping if it symbol is bounded. Therefore, we can use the norm in $C_{0}\left(S^{d-1}\right)$ instead of the norm in $C^{d}\left(S^{d-1}\right)$. We then have the following theorem.

Theorem 2.2. Let $\left(u_{n}\right)$ be a bounded sequence in $L^{p}\left(\mathbf{R}^{d+m}\right), p \geq 2$, and let $\left(v_{n}\right)$ be a uniformly compactly supported bounded sequence of functions converging weakly to zero in $L^{q}\left(\mathbf{R}^{d}\right)$ for some (finite) $q>p^{\prime}$. Let $r \in(1, p)$ be such that $\frac{1}{p}+\frac{1}{r^{\prime}}+\frac{1}{q}=1$. Then, after passing to a subsequence (not relabeled), there exists a continuous bilinear functional $\mu$ on $L^{r}\left(\mathbf{R}^{d}\right) \otimes L^{p^{\prime}}\left(\mathbf{R}^{m} ; C\left(S^{d-1}\right)\right)$ 
such that for every $\phi \in L^{r}\left(\mathbf{R}^{d}\right)$ and $\psi \in L^{p^{\prime}}\left(\mathbf{R}^{m} ; C\left(S^{d-1}\right)\right)$, it holds

$$
\mu(\phi, \bar{\psi})=\lim _{n \rightarrow \infty} \int_{\mathbf{R}^{d+m}} \phi(\mathbf{x}) u_{n}(\mathbf{x}, \lambda) \overline{\mathcal{A}_{\psi(\lambda, \boldsymbol{\xi} /|\boldsymbol{\xi}|)}\left(v_{n}\right)(\mathbf{x})} d \mathbf{x} d \lambda
$$

where $\mathcal{A}_{\psi(\lambda, \boldsymbol{\xi} /|\boldsymbol{\xi}|)}$ is the Fourier multiplier operator on $\mathbf{R}^{d}$ with the symbol $\psi(\lambda, \boldsymbol{\xi} /|\boldsymbol{\xi}|)$.

The functional $\mu$ is called the H-measure corresponding to (sub)sequences (of) $\left(u_{n}\right)$ and $\left(v_{n}\right)$. It extends continuously to $\tilde{W}_{0}^{(\bar{s}, s)}$.

\section{Proof of Theorem 1.2}

We are now ready to prove the main theorem. To this end, take the family of the mollifiers $\omega_{\delta}$ given in Definition 1.1 and consider the following family of approximations to (1.1) with the initial data $u_{0}$ :

$$
\partial_{t} u_{\delta}+\operatorname{div}_{\mathbf{x}} \mathfrak{f}_{\delta}\left(\mathbf{x}, u_{\delta}\right)=0
$$

for $\mathfrak{f}_{\delta}=\mathfrak{f} \star \frac{1}{\delta} \omega_{\delta}(\mathbf{x} / \delta)$ where $\star$ denotes the convolution operator. Since the flux $\mathfrak{f}_{\delta}$ is smooth, there exists an entropy admissible solution to (3.1) in the standard Kruzhkov sense ([13]), $\left.u_{\delta}\right|_{t=0}=u_{0}(\mathbf{x})$ satisfying for any $\lambda \in \mathbf{R}$ :

$$
\begin{aligned}
\partial_{t} \mid u_{\delta} & -\lambda \mid+\operatorname{div}_{\mathbf{x}}\left(\operatorname{sgn}\left(u_{\delta}-\lambda\right)\left(\mathfrak{f}_{\delta}\left(\mathbf{x}, u_{\delta}\right)-\mathfrak{f}_{\delta}(\mathbf{x}, \lambda)\right)\right) \\
& \leq \operatorname{sgn}\left(u_{\delta}-\lambda\right) \operatorname{div}_{\mathbf{x}} \mathfrak{f}_{\delta}(\mathbf{x}, \lambda) .
\end{aligned}
$$

Moreover, since $\mathfrak{f}_{\delta}(\mathbf{x}, 2 a)=\mathfrak{f}_{\delta}(\mathbf{x}, 2 b)=0$ for $\delta$ small enough, the family of solutions $\left(u_{\delta}\right)$ will remain bounded between $2 a$ and $2 b$ (see e.g. [23]).

We fix $\delta_{0}>0$ and, using also Schwartz lemma on non-negative distributions, we rewrite the equation in the form

$$
\begin{aligned}
\partial_{t} \mid u_{\delta}- & \lambda \mid+\operatorname{div}_{\mathbf{x}}\left(\operatorname{sgn}\left(u_{\delta}-\lambda\right)\left(\mathfrak{f}_{\delta_{0}}\left(\mathbf{x}, u_{\delta}\right)-\mathfrak{f}_{\delta_{0}}(\mathbf{x}, \lambda)\right)\right) \\
= & \operatorname{div}_{\mathbf{x}}\left(\operatorname{sgn}\left(u_{\delta}-\lambda\right)\left(\left(\mathfrak{f}_{\delta_{0}}-\mathfrak{f}_{\delta}\right)\left(\mathbf{x}, u_{\delta}\right)-\left(\mathfrak{f}_{\delta_{0}}-\mathfrak{f}_{\delta}\right)(\mathbf{x}, \lambda)\right)\right) \\
& +\operatorname{sgn}\left(u_{\delta}-\lambda\right) \operatorname{div}_{\mathbf{x}} \mathfrak{f}_{\delta}(\mathbf{x}, \lambda)-m_{\delta}(t, \mathbf{x}, \lambda),
\end{aligned}
$$

for a non-negative Radon measure $m_{\delta} \in \mathcal{M}\left(\mathbf{R}^{+} \times \mathbf{R}^{d} \times \mathbf{R}\right)$.

Then, by finding derivative of the such expression with respect to $\lambda$, we get

$$
\begin{aligned}
\partial_{t} h_{\delta}(t, \mathbf{x}, \lambda)+\operatorname{div}_{\mathbf{x}}\left(\mathfrak{f}_{\delta_{0}}^{\prime}(\mathbf{x}, \lambda) h_{\delta}(t, \mathbf{x}, \lambda)\right) & \\
= & \partial_{\lambda} \operatorname{div}_{\mathbf{x}}\left(\operatorname{sgn}\left(u_{\delta}-\lambda\right)\left(\left(\mathfrak{f}_{\delta_{0}}-\mathfrak{f}_{\delta}\right)\left(\mathbf{x}, u_{\delta}\right)-\left(\mathfrak{f}_{\delta_{0}}-\mathfrak{f}_{\delta}\right)(\mathbf{x}, \lambda)\right)\right) \\
& +\partial_{\lambda}\left(\operatorname{sgn}\left(u_{\delta}-\lambda\right) \operatorname{div}_{\mathbf{x}} \mathfrak{f}_{\delta}(\mathbf{x}, \lambda)\right)+\partial_{\lambda} m_{\delta}(t, \mathbf{x}, \lambda),
\end{aligned}
$$

where $\mathfrak{f}_{\delta_{0}}^{\prime}(\mathbf{x}, \lambda)=\partial_{\lambda} \mathfrak{f}_{\delta_{0}}(\mathbf{x}, \lambda)$ and $h_{\delta}(t, \mathbf{x}, \lambda)=\partial_{\lambda}\left|u_{\delta}-\lambda\right|$.

As usual when applying the micro-local defect functionals, we first need to derive the localization principle from equation (3.3). The localization principle describes the support of the corresponding micro-local defect functional. To 
this end, for fixed $\rho \in C_{c}\left(\mathbf{R}^{m}\right)$ and $\tilde{\varphi} \in C_{c}\left(\mathbf{R}_{+}^{d}\right)$, denote by $V$ a weak-* $L^{\infty}\left(\mathbf{R}_{+}^{d}\right)$ limit along some not relabelled subsequence of the sequence

$$
V_{\delta}= \begin{cases}\frac{\tilde{\varphi}(t, \mathbf{x}) \int_{\mathbf{R}^{m}} \rho(\lambda) h_{\delta}(t, \mathbf{x}, \lambda) d \lambda}{\left|\int_{\mathbf{R}^{m}} \rho(\lambda) h_{\delta}(t, \mathbf{x}, \lambda) d \lambda\right|}, & \int_{\mathbf{R}^{m}} \rho(\lambda) h_{\delta}(t, \mathbf{x}, \lambda) d \lambda \neq 0, \\ 0, & \text { otherwise. }\end{cases}
$$

Denote $v_{\delta}=V_{\delta}-V$ and note that the subsequence of the family $\left(v_{\delta}\right)$ satisfies the conditions of Theorem 2.1 and Theorem 2.2.

Denote

$$
U_{\delta}^{\delta_{0}}=\operatorname{sgn}\left(u_{\delta}-\lambda\right)\left(\left(\mathfrak{f}_{\delta_{0}}-\mathfrak{f}_{\delta}\right)\left(\mathbf{x}, u_{\delta}\right)-\left(\mathfrak{f}_{\delta_{0}}-\mathfrak{f}_{\delta}\right)(\mathbf{x}, \lambda)\right),
$$

where $\tilde{\varphi} \in C_{c}^{1}([0, T] \times \Omega)$ for a fixed $T>0$ and $\Omega \subset \subset \mathbf{R}^{d}$.

Due to linearity, we can assume without losing on generality that $h_{\delta} \stackrel{*}{\rightarrow} 0$ in $L^{\infty}\left(\mathbf{R}_{+}^{d} \times \mathbf{R}\right)$. Remark that this implies $m_{\delta} \rightarrow 0$ in the space of Radon measures. We then denote by

(HM) $\mu$-the $\mathrm{H}$-measure corresponding to a subsequence of the families $\left(h_{\delta}\right)$ and $\left(v_{\delta}\right)$;

(HD) $\mu_{\delta_{0}}$ - the $\mathrm{H}$-distribution corresponding to a subsequence of the families $\left(U_{\delta}^{\delta_{0}}\right)$ and $\left(v_{\delta}\right)$.

We can assume that both $\mu$ and $\mu_{\delta_{0}}$ are defined along the same subsequences. According to Lemma A.2, we know that

$$
\left\|\mu_{\delta_{0}}\right\| \lesssim \tilde{\sigma}\left(\delta_{0}\right)
$$

where $\tilde{\sigma}\left(\delta_{0}\right) \rightarrow 0$ as $\delta_{0} \rightarrow 0$.

Now, we take the test function of the form

$$
\phi_{n}(t, \mathbf{x}, \lambda)=\varphi_{1}(t, \mathbf{x}) \rho_{1}(\lambda)\left(\mathcal{T}_{-1} \circ \mathcal{A}_{\psi((\tau, \boldsymbol{\xi}) /|(\tau, \boldsymbol{\xi})|)}\left(v_{n}\right)\right)(t, \mathbf{x}),
$$

where $\mathcal{T}_{-1}$ is the Riesz transform ([11]), i.e. the multiplier operator with the symbol $\frac{1}{(\tau, \boldsymbol{\xi}))}$, which, as is well known, maps continuously $L^{p}\left(\mathbf{R}^{d}\right) \rightarrow$ $W^{1, p}\left(\mathbf{R}^{d}\right)$. As for the other functions, we have $\psi \in C^{d}\left(S^{d}\right), \varphi_{1} \in C_{c}^{1}\left(\mathbf{R}^{d}\right)$, $\rho_{1} \in C_{c}^{1}\left(\mathbf{R}^{m}\right)$. We test (3.3) with respect to the $\phi_{n}$ and then let $n \rightarrow \infty$ along the subsequence defining the $\mathrm{H}$-measure $\mu$ and the $\mathrm{H}$-distribution $\mu_{\delta_{0}}$. We get (see e.g. [16, Theorem 3.4]):

$$
\begin{aligned}
& \left.\left\langle\varphi_{1}(t, \mathbf{x}) \rho_{1}(\lambda) \psi(\tau, \boldsymbol{\xi})\left(\tau+\left\langle\mathfrak{f}_{\delta_{0}}(\mathbf{x}, \lambda), \boldsymbol{\xi}\right\rangle\right\rangle\right), \mu\right\rangle \\
& =\left\langle\varphi_{1}(t, \mathbf{x}) \tilde{\varphi}(t, \mathbf{x}) \rho_{1}^{\prime}(\lambda) \psi(\tau, \boldsymbol{\xi}) \sum_{k=1}^{d} \xi_{j}, \mu_{\delta_{0}}\right\rangle .
\end{aligned}
$$

According to the density arguments, we can replace $\varphi_{1}(t, \mathbf{x}) \rho_{1}(\lambda) \psi(\tau, \boldsymbol{\xi})$ by an arbitrary $\phi \in W^{(p, \tilde{p})}$. Having this in mind, we take the following test function to substitute in (3.6) instead of $\varphi_{1} \rho_{1} \psi$ :

$$
\phi_{\delta_{0}}(t, \mathbf{x}, \lambda, \tau, \boldsymbol{\xi})=\varphi(t, \mathbf{x}) \rho(\lambda) \psi(\tau, \boldsymbol{\xi}) \frac{\left(\tau+\left\langle\mathfrak{f}_{\tilde{\delta}}(\mathbf{x}, \lambda), \boldsymbol{\xi}\right\rangle\right)}{\left.\left|\tau+\left\langle\mathfrak{f}_{\tilde{\delta}}(\mathbf{x}, \lambda), \boldsymbol{\xi}\right\rangle\right\rangle\right|^{2}+\sigma\left(\delta_{0}\right)}
$$


where $\varphi$ is an arbitrary $C_{c}\left(\mathbf{R}_{+}^{d}\right)$-function, and we choose

$$
\tilde{\delta}\left(\delta_{0}\right) \gg \delta_{0} \text { and } \sigma\left(\delta_{0}\right) \gg \delta_{0}
$$

such that, for $\tilde{\sigma}\left(\delta_{0}\right)$ from (3.5), we have

$$
\tilde{\sigma}\left(\delta_{0}\right)\left\|\partial_{\lambda} \phi_{\delta_{0}}\right\|_{W^{(p, \tilde{p})}} \rightarrow 0 .
$$

Such a choice is clearly possible by taking for instance $\tilde{\delta}\left(\delta_{0}\right)=\exp \left(-\frac{1}{\tilde{\sigma}\left(\delta_{0}\right)}\right)$, taking into account (3.5) and definition of $W^{(p, \tilde{p})}$. Substituting the test function $\phi_{\delta_{0}}$ into (3.6), according to Lemma A.1 and Lemma A.2, we get after letting $\delta_{0} \rightarrow 0$

$$
\langle\varphi \rho, \mu\rangle=0 .
$$

Since $\varphi$ is arbitrary, we conclude that the (weighted) H-measure $\rho \mu$ is zero and thus, the family $\left(\int \rho(\lambda) h_{\delta} d \lambda\right)$ contains an $L_{l o c}^{1}$-strongly convergent subsequence. Using the density argument, we can choose $\rho(\lambda)=\chi_{[-M, M]}(\lambda)$ for the characteristic function $\chi_{[-M, M]}$ of the interval $[-M, M]$. Thus we get

$$
\int \rho(\lambda) h_{\delta} d \lambda=\int_{-M}^{M} \operatorname{sgn}\left(u_{\delta}-\lambda\right) d \lambda=2 u_{\delta}
$$

implying that $\left(u_{\delta}\right)$ is $L_{l o c}^{1}$-strongly precompact. The strong $L_{l o c}^{1}$-limit along any subsequence is the solution to the considered problem (1.1), $\left.u\right|_{t=0}=$ $u_{0}(\mathbf{x})$.

\section{Appendix A.}

LEMmA A.1. Assume that the flux $\mathfrak{f}$ from (1.1) satisfies the nondegeneracy conditions. Then, for any $\phi \in L_{c}^{p}\left(\mathbf{R}_{+}^{d+1} ; C^{d}\left(S^{d}\right)\right)$ and $\rho$ defined in (3.4) the following holds:

$$
\liminf _{\delta_{0} \rightarrow 0}\left\langle\phi \rho \frac{\left(\tau+\left\langle\mathfrak{f}_{\delta_{0}}^{\prime}(\mathbf{x}, \lambda), \boldsymbol{\xi}\right\rangle\right) \cdot\left(\tau+\left\langle\mathfrak{f}_{\delta}^{\prime}(\mathbf{x}, \lambda), \boldsymbol{\xi}\right\rangle\right)}{\left|\tau+\left\langle\mathfrak{f}_{\delta}^{\prime}(\mathbf{x}, \lambda), \boldsymbol{\xi}\right\rangle\right|^{2}+\sigma\left(\delta_{0}\right)}, \mu\right\rangle=\langle\phi \rho, \mu\rangle,
$$

where $\mu$ is the H-measure defined in HM.

Proof. We can rewrite (A.1) in the form

$$
\begin{aligned}
& \langle\phi \rho, \mu\rangle+\liminf _{\delta_{0} \rightarrow 0}\left\langle\phi \rho\left(\frac{\left(\tau+\left\langle\mathfrak{f}_{\delta_{0}}^{\prime}(\mathbf{x}, \lambda), \boldsymbol{\xi}\right\rangle\right) \cdot\left(\tau+\left\langle\mathfrak{f}_{\delta}^{\prime}(\mathbf{x}, \lambda), \boldsymbol{\xi}\right\rangle\right)}{\left|\tau+\left\langle\mathfrak{f}_{\delta}^{\prime}(\mathbf{x}, \lambda), \boldsymbol{\xi}\right\rangle\right|^{2}+\sigma\left(\delta_{0}\right)}-1\right), \mu\right\rangle \\
& \quad=\langle\phi \rho, \mu\rangle
\end{aligned}
$$

which is clearly correct since the second term on the right-hand side of the latter expression tends to zero according to the non-degeneracy conditions 
and Theorem 2.2:

$$
\begin{aligned}
& \left|\left\langle\phi \rho\left(\frac{\left(\tau+\left\langle\mathfrak{f}_{\delta_{0}}^{\prime}(\mathbf{x}, \lambda), \boldsymbol{\xi}\right\rangle\right) \cdot\left(\tau+\left\langle\mathfrak{f}_{\delta}^{\prime}(\mathbf{x}, \lambda), \boldsymbol{\xi}\right\rangle\right)}{\left|\tau+\left\langle\mathfrak{f}_{\delta}^{\prime}(\mathbf{x}, \lambda), \boldsymbol{\xi}\right\rangle\right|^{2}+\sigma\left(\delta_{0}\right)}-1\right), \mu\right\rangle\right| \\
& \quad \leq\left\|\phi \rho\left(\frac{\left(\tau+\left\langle\mathfrak{f}_{\delta_{0}}^{\prime}(\mathbf{x}, \lambda), \boldsymbol{\xi}\right\rangle\right) \cdot\left(\tau+\left\langle\mathfrak{f}_{\delta}^{\prime}(\mathbf{x}, \lambda), \boldsymbol{\xi}\right\rangle\right)}{\left|\tau+\left\langle\mathfrak{f}_{\delta}^{\prime}(\mathbf{x}, \lambda), \boldsymbol{\xi}\right\rangle\right|^{2}+\sigma\left(\delta_{0}\right)}-1\right)\right\|_{W_{0}^{2, \tilde{z}}\|\mu\| .}
\end{aligned}
$$

Having in mind definition of the norm $\|\cdot\|_{W_{0}^{p, \tilde{p}}}$ and using the Lebesgue dominated convergence theorem, we conclude the lemma.

Lemma A.2. Under the assumptions $\left|u_{\delta}\right| \leq M$, it holds (for $\delta$ small enough)

$$
\left\|\mu_{\delta}\right\| \lesssim\left\|\sup _{\lambda, \eta \in[-M, M]} \mid \mathfrak{f}(\mathbf{x}, \lambda+\delta \eta)-\mathfrak{f}(\mathbf{x}, \lambda)\right\|_{L^{p}(\Omega)}=\tilde{\sigma}(\delta) \rightarrow 0
$$

as $\delta \rightarrow 0$.

Proof. From [17], we know that the bound of the H-distribution $\mu_{\delta_{0}}$ equals (for the interval $I \subset[-M, M]$ where $\rho$ from (3.4) is supported)

$$
\begin{aligned}
\left\|\mu_{\delta_{0}}\right\| \leq & C \| \tilde{\varphi}_{1} \operatorname{sgn}\left(u_{\delta}-\lambda\right)\left(\left(\mathfrak{f}_{\delta_{0}}-\mathfrak{f}_{\delta}\right)\left(\mathbf{x}, u_{\delta}\right)-\left(\mathfrak{f}_{\delta_{0}}-\mathfrak{f}_{\delta}\right)(\mathbf{x}, \lambda) \|_{L^{p}([0, T) \times \Omega \times I)}\right. \\
& \left\|v_{\delta}\right\|_{L^{\tilde{p}}([0, T) \times \Omega)} .
\end{aligned}
$$

Clearly, according to the definition of the convolution, we have

$$
\begin{gathered}
\| \tilde{\varphi}_{1} \operatorname{sgn}\left(u_{\delta}-\lambda\right)\left(\left(\mathfrak{f}_{\delta_{0}}-\mathfrak{f}_{\delta}\right)\left(\mathbf{x}, u_{\delta}\right)-\left(\mathfrak{f}_{\delta_{0}}-\mathfrak{f}_{\delta}\right)(\mathbf{x}, \lambda) \|_{L^{p}([0, T) \times \Omega \times I)}\right. \\
\leq C(T)\left\|\sup _{\lambda, \eta \in[-M, M]}|\mathfrak{f}(\mathbf{x}, \lambda+\delta \eta)-\mathfrak{f}(\mathbf{x}, \lambda)|\right\|_{L^{p}(\Omega)} .
\end{gathered}
$$

Since $\mathfrak{f}$ is a continuous function with respect to $\lambda$, we know that for almost every $\mathbf{x} \in \Omega$, it must hold

$$
\sup _{\lambda, \eta \in[-M, M]}|\mathfrak{f}(\mathbf{x}, \lambda+\delta \eta)-\mathfrak{f}(\mathbf{x}, \lambda)| \rightarrow 0
$$

since a continuous function on a compact set is uniformly continuous as well. Now, from the Lebesgue dominated convergence theorem, we conclude (A.2).

\section{ACKNowledgements.}

The paper was partly written while the author was the guest of the University of Vienna in the frame of the FWF project M-2669 Meitner-Programme whose support we gratefully acknowledge. 


\section{REFERENCES}

[1] Adimurthi, S. Mishra and G.D.V. Gowda, Optimal entropy solutions for conservation laws with discontinuous flux, J.Hyperbolic Differ. Equ. 2 (2005), 783-837.

[2] B. Andreianov and D. Mitrovic, Entropy conditions for scalar conservation laws with discontinuous flux revisited, Ann. Inst. H. Poincaré Anal. Non Linéaire 32 (2015), 1307-1335.

[3] B. Andreianov, K. H. Karlsen and N. H. Risebro, A theory of $L^{1}$-dissipative solvers for scalar conservation laws with discontinuous flux, Arch. Ration. Mech. Anal. 201 (2011), 27-86.

[4] N. Antonić, M. Erceg and M. Lazar, Localisation principle for one-scale H-measures, J. Funct. Anal. 272 (2017), 3410-3454.

[5] N. Antonić and M. Lazar, Parabolic H-measures, J. Funct. Anal. 265 (2013), 11901239.

[6] N. Antonić and D. Mitrović, $H$-distributions, an extension of $H$-measures to an $l^{p}-l^{q}$ setting, Abstr. Appl. Anal. 2011, Article ID 901084, 12 pp.

[7] R. Bürger, K. H. Karlsen, H. Torres and J. Towers, Second-order schemes for conservation laws with discontinuous flux modelling clarifier-thickener units, Numer. Math. 116 (2010), 579-617.

[8] R. Colombo, K. H. Karlsen, F. Lagoutiere and A. Marson, Special issue on contemporary topics in conservation laws, Netw. Heterog. Media 11 (2016), i-ii.

[9] P. Gérard, Microlocal defect measures, Comm. Partial Differential Equations 16 (1991), 1761-1794.

[10] M. Garavello, R. Natalini, B. Piccoli and A. Terracina, Conservation laws with discontinuous flux, Netw. Heter. Media 2 (2007), 159-179.

[11] L. Grafakos, Classical Fourier analysis, Springer, New York, 2008.

[12] K. H. Karlsen, N. H. Risebro and J. Towers, $L^{1}$-stability for entropy solutions of nonlinear degenerate parabolic convection-diffusion equations with discontinuous coefficients, Skr. K. Nor. Vidensk. Selsk. 3 (2003), 1-49.

[13] S. N. Kruzhkov, First order quasilinear equations with several independent variables, Mat. Sb. (N.S.) 81(123) (1970), 228-255.

[14] M. Lazar and D. Mitrović, Velocity averaging - a general framework, Dynamics of PDEs 9 (2012) 239-260.

[15] M. Lazar and D. Mitrović, On an extension of a bilinear functional on $L^{p}\left(\mathbf{R}^{n}\right) \times E$ to Bôchner spaces with an application to velocity averaging, C. R. Math. Acad. Sci. Paris 351 (2013), 261-264.

[16] M. Lazar and D. Mitrović, Existence of solutions for a scalar conservation law with a flux of low regularity, Electron. J. Differential Equations 2016, No. 325, 18 pp.

[17] M. Lazar and D. Mitrović, On a new class of functional spaces with application to the velocity averaging, Glas. Mat. Ser. III 52(72) (2017), 115-130.

[18] P.L. Lions, B. Perthame and E. Tadmor, A kinetic formulation of multidimensional scalar conservation law and related equations, J. Amer. Math. Soc. 7 (1994), 169-191.

[19] M. Mišur and D. Mitrović, On a generalization of compensated compactness in the $L^{p}-L^{q}$ setting, J. Funct. Anal. 268 (2015), 1904-1927.

[20] W. Neves, E.Yu. Panov and J. Silva, Strong traces for conservation laws with general nonautonomous flux, SIAM J. Math. Anal. 50 (2018), 6049-6081.

[21] E. Yu. Panov, Generalized solutions of the Cauchy problem for quasilinear conservation laws, Dissertation Can. Phys.-Math. Sci., Mosk. Gos. Univ., Moscow, 1991 (Russian).

[22] E.Yu. Panov, On existence and uniqueness of entropy solutions to the Cauchy problem for a conservation law with discontinuous flux, J. Hyperbolic Differ. Equ. 6 (2009), $525-548$. 
[23] E.Yu. Panov, Existence and strong pre-compactness properties for entropy solutions of a first-order quasilinear equation with discontinuous flux, Arch. Ration. Mech. Anal. 195 (2010), 643-673.

[24] E.Yu. Panov, Ultra-parabolic H-measures and compensated compactness, Ann. Inst. H. Poincaré Anal. Non Linéaire 28 (2011), 47-62.

[25] F. Rindler, Directional oscillations, concentrations, and compensated compactness via microlocal compactness forms, Arch. Ration. Mech. Anal. 215 (2015), 1-63.

[26] L. Tartar, H-measures, a new approach for studying homogenisation, oscillations and concentration effects in partial differential equations, Proc. Roy. Soc. Edinburgh Sect. A 115 (1990), 193-230.

[27] L. Tartar, Multi-scales H-measures, Discrete Contin. Dyn. Syst. Ser. S 8 (2015) 77-90.

[28] B. Temple, Global solution of the Cauchy problem for a class of $2 \times 2$ nonstrictly hyperbolic conservation laws, Adv. in Appl. Math. 3 (1982), 335-375.

N. Konatar

Department of Natural Sciences and Mathematics

Faculty of Mathematics and Natural Sciences

University of Montenegro

Džordža Vašingtona bb, 81000 Podgorica

Crna Gora

E-mail: nikola.k@ucg.ac.me

Received: 25.7.2019.

Revised: 6.10 .2019 . 